\title{
Measuring the Intraday Jump Tail Risk of Financial Asset Price with Noisy High Frequency Data
}

\author{
Chao Yu' ${ }^{1}$ Xujie Zhao², Feng Zhang1 \\ ${ }^{1}$ School of Statistics, University of International Business and Economics, Beijing, China \\ ${ }^{2}$ School of International Trade and Economics, University of International Business and Economics, Beijing, China \\ Email: chaoyu@uibe.edu.cn
}

How to cite this paper: Yu, C., Zhao, X.J. and Zhang, F. (2017) Measuring the Intraday Jump Tail Risk of Financial Asset Price with Noisy High Frequency Data. Open Journal of Statistics, 7, 72-83.

https://doi.org/10.4236/ojs.2017.71006

Received: December 28, 2016

Accepted: February 17, 2017

Published: February 20, 2017

Copyright $\odot 2017$ by authors and Scientific Research Publishing Inc. This work is licensed under the Creative Commons Attribution International License (CC BY 4.0).

http://creativecommons.org/licenses/by/4.0/

\begin{abstract}
This paper proposes a simple two-step nonparametric procedure to estimate the intraday jump tail and measure the jump tail risk in asset price with noisy high frequency data. We first propose the pre-averaging threshold approach to estimate the intraday jumps occurred, and then use the peaks-overthreshold (POT) method and generalized Pareto distribution (GPD) to model the intraday jump tail and further measure the jump tail risk. Finally, an empirical example further demonstrates the power of the proposed method to measure the jump tail risk under the effect of microstructure noise.
\end{abstract}

\section{Keywords}

High Frequency Data, Intraday Jump, Microstructure Noise, Jump Tail Risk, Pre-Averaging

\section{Introduction}

It's well recognized that the financial asset returns are not normally distributed, but instead exhibit more slowly decaying and asymmetric tails. The earliest influential researches in Mandelbrot [1] and Fama [2] show the empirical evidence for fat-tailed return distributions. And the numerous subsequent studies show that these fatter tails may be attributable to stochastic volatility and/or occasionally large absolute price changes, called "jumps" in the underlying asset price process. With the availability of reliable financial high frequency data over the last two decades, many closer researches on the dynamics of financial asset prices have documented the presence of jumps; see Barndorff-Nielsen and Shephard 
[3] [4], Huangand Tauchen [5], Aït-Sahalia and Jacod [6], Lee and Hannig [7], Lee and Mykland [8], and so on. While both components can account for the extreme tail behavior, they have different mechanisms and further have very different implications on pricing and risk management, as recently explored by Bollerslev and Todorov [9].

In contrast to the numerous studies on tail risk resulting from stochastic volatility, there is fewer work to study the jump tail risk. To the best of our knowledge, recent contributions are mainly from Bollerslev and Todorov [9] [10] [11]. However, the recent financial crisis has further spurred the interest of studying the jump tail events, and the econometric techniques for more accurately estimating and modeling such risks. On the other hand, the existing studies on jump tail risk are performed under the assumption of semimartingale price process in an idealized world. The real application, however, runs into wellknown bias problem caused by market microstructure noise, when the data frequency is very high. The presence of market microstructure noise is widely demonstrated in literature; see O'Hara [12], Hasbrouck [13] and the references therein. Such kind of noises are usually caused by the frictions in actual trades, including tick size, discrete observation, bid-ask spread, and other trading mechanics. Hence, how to estimate the jump tails and measure the jump tail risk under the effect of microstructure noise is of great significance in real application. Although there are some methods proposed to deal with the noise, such as the two time scale and multi-time scale approach (Zhang, et al. [14] [15]), pre-averaging method (Podolskij and Vetter [16], Jacod, Li, Mykland [17]) and realized kernel method (Barndorff-Nielsen and Shephard [18]), most of them are used in the scenarios of estimating the integrated volatility or testing the jump component. In this paper, we consider the problem of estimating the jump tail and measuring the jump tail risk when the observations are contaminated by microstructure noise.

In this paper, we focus on studying the intraday jump tail and measuring the jump tail risk under the market microstructure noise. A simple two-step nonparametric procedure is proposed to implement the analysis. In first step, we use the pre-averaging threshold method to nonparametrically estimate the intraday jump under the effect of microstructure noise. In particular, we first adopt local "pre-averaging" via a kernel function to produce a set of non-overlapping (asymptotically) noise-free observations, and then use the threshold technique to identify the jump series. In second step, we model the intraday jump tail based on the extreme value theory (EVT) and further calculate the jump tail risk measure (Value-at-Risk and Expected Shortfall). Our method is nonparametric, and is easy to implement. Finally, a real data example with actual high frequency data of MSFT is used to show these procedures.

The remainder of this paper is organized as follows. Section 2 presents the methodology to estimate the intraday jump and jump tail risk measurement. Section 3 provides an empirical example to show the procedure. Section 4 draws conclusions. 


\section{Intraday Jump Tail Risk Measurement under Microstructure Noise}

In this section, a simple two-step procedure is proposed to measure the intraday jump tail risk with noisy high frequency data. In first step, a pre-averaging threshold method is proposed to nonparametrically identify the intraday jump under the effect of microstructure noise. In second step, the peaks-over-threshold (POT) method based on the generalized Pareto distribution (GPD) is used to model the intraday jump tail and further to calculate the jump tail risk measure, i.e. VaR (Value-at-Risk) and ES (Expected Shortfall).

\subsection{Pre-Averaging Threshold Estimation of Intraday Jump}

Assume that the efficient logarithmic price $p_{t}$ of an asset defined on a filtered probability space $\left(\Omega, \mathcal{F},\left(\mathcal{F}_{t}\right)_{t \geq 0}, \mathbb{P}\right)$, evolves as

$$
\mathrm{d} p_{t}=b_{t} \mathrm{~d} t+\sigma_{t} \mathrm{~d} W_{t}+\mathrm{d} J_{t},
$$

where $W=\left(W_{t}\right)$ is an $\mathcal{F}$-adapted standard Brownian motion. The drift $b=\left(b_{t}\right)$ and the volatility $\sigma=\left(\sigma_{t}\right)$ are progressively measurable processes which guarantee that (1) has a unique, strong solution, which are adapted and right continuous with left limits (càdlàg) processes. $J=\left(J_{t}\right)$ is a compound Poisson process with finite activity of jumps. Note that $J_{t}$ can be written as $J_{t}=\sum_{i=1}^{N_{t}} X_{\tau_{i}}$, where $\left(N_{t}\right)$ is a Poisson process with intensity $\lambda$, and $X_{\tau_{i}}$ denotes the jump size at the jump location $\tau_{i} . X_{\tau_{i}}$ are independent identically distributed and independent of $N_{t}$. We further assume that $N_{t}$ is independent of $W_{t}$. However, our results can extend to the scenarios with non-constant intensity and more general dependence structure between $X_{\tau_{i}}$ and $N_{t}$.

Suppose that on the finite and fixed time horizon $[0, T]$, there are $n+1$ discrete realization $p_{t_{0}}, p_{t_{1}}, \cdots, p_{t_{n-1}}, p_{t_{n}}$ of process $p_{t} \cdot 0=t_{0}<t_{1}<\cdots<t_{n}=T$ is an arbitrary partition of interval $[0, T]$. For simplicity, assume that the observations are equally spaced. Denote $\Delta_{n}=T / n$, then $t_{i}=i \Delta_{n}$. In the presence of microstructure noise, at any given time $t_{i}$, the actually observed log-price is $Z_{t_{i}}$ other than $p_{t_{i}}$, which can be given as

$$
Z_{t_{i}}=p_{t_{i}}+\varepsilon_{t_{i}},
$$

where $\varepsilon_{t}$ is the noise term. Assume that the $\varepsilon_{t}$ s are i.i.d. and independent of $W_{t}$ and $J_{t}$ processes, and with $E \varepsilon_{t}=0$, and $E \varepsilon_{t}^{2}<\infty$. Although the noises are not necessary i.i.d, this assumption is only for the simplicity to prove the theoretical properties. See the studies in Yu et al. [19], where we show that the estimation method for intraday jump used in this paper also performs well in the setting of correlated noises.

Our goal is to estimate the intraday jump $X_{i}$, with these noisy observation data $\left\{Z_{t_{i}}, i=0,1, \cdots, n\right\}$. For the simplicity of notation, we denote $V_{i}^{n}=V_{i \Delta_{n}}$, $\Delta_{i}^{n} V=V_{i}^{n}-V_{i-1}^{n}$ for any process $V=\left(V_{t}\right)$ in the following.

In this paper, we use the pre-averaging approach to diminish the effect of noise. Let $\bar{Z}_{i}^{n}$ denote the weighted average of $k_{n}$ observations of 
$Z_{i}^{n}, Z_{i+1}^{n}, \cdots, Z_{i+k_{n}-1}^{n}$, where $\bar{Z}_{i}^{n}=\sum_{j=1}^{k_{n}-1} g_{j}^{n} \Delta_{i+j}^{n} Z$, with weights $g_{j}^{n}=g\left(j / k_{n}\right)$. We require that the weighting function $g(x)$ is continuous on $[0,1]$, piecewise $C^{1}$ with a piecewise Lipschitz derivative $g^{\prime}$, and satisfies $g(0)=g(1)=0$, $\int_{0}^{1} g(s)^{2} \mathrm{~d} s>0$. We further require that the integer sequence $k_{n}$ satisfies $k_{n} \sqrt{\Delta_{n}}=\theta+o\left(\Delta_{n}^{1 / 4}\right)$ for some constant $\theta>0$.

Then we can use the threshold technique to identify the jump with these pre-averaging observations $\left\{\bar{Z}_{i}^{n}\right\}$. The threshold function is required to satisfy the following assumption.

Assumption 1 The threshold function $r\left(\Delta_{n}\right)$ is a deterministic function of the step length $\Delta_{n}$, such that

(a) $\lim _{\Delta_{n} \rightarrow 0} r\left(\Delta_{n}\right)=0$;

(b) $\lim _{\Delta_{n} \rightarrow 0} \frac{\Delta_{n}^{1 / 2}\left(\log \frac{1}{\Delta_{n}}\right)^{2}}{r\left(\Delta_{n}\right)}=0$.

Power functions $r\left(\Delta_{n}\right)=\beta \Delta_{n}^{\alpha}$ for any $\alpha \in(0,1 / 2)$ and $\beta \in R$ are possible choices. Under the Assumption 1, for $P$-almost all $\omega, \exists \Delta>0$ such that $\forall \Delta_{n} \leq \Delta$, we have that $\forall i=0, \cdots, n-1, \quad I_{\left\{\left(\bar{Z}_{i}^{n}\right)^{2} \leq r\left(\Delta_{n}\right)\right\}}=I_{\left\{n_{\substack{i+k_{j}-1 \\ j=1+1}}\left(\Delta_{j} N=0\right)\right\}}$, which says that the threshold function $r\left(\Delta_{n}\right)$ can be used to asymptotically identify the intervals where no jump occurred; also see the literature on the noise- and jumprobust volatility estimation (Jing et al. [20]). In other words, if $\left(\bar{Z}_{i}^{n}\right)^{2} \leq r\left(\Delta_{n}\right)$, there exists jumps on interval $\left(t_{i}, t_{i+k_{n}-1}\right]$. Thus, we can use this threshold method to identify the intervals where jump occurs and further give a coarse estimation of the location of jumps. Let $T_{\tau}$ denote the location set of jumps occurred on $[0, T]$, then

$$
\hat{T}_{\tau}=\left\{t_{i *} \in[0, T]:\left(\bar{Z}_{i *}^{n}\right)^{2}>r\left(\Delta_{n}\right), i=0, \cdots, j_{n}\right\},
$$

where $i *=i\left(k_{n}-1\right)$.

We now turn to estimate the jump size by a simple nonparametric method. Denote by $\Delta_{i}^{i+k_{n}-1} N=N_{\left(i+k_{n}-1\right) \Delta_{n}}-N_{i \Delta_{n}}$; if $\Delta_{i}^{i+k_{n}-1} N \geq 1$, by $\tau^{(i)}$ the first instant a jump occurs within $\left(t_{i}, t_{i+k_{n}-1}\right]$, and $X_{\tau^{(i)}}$ the size of this first jump, also let $\bar{X}=\min _{j=1, \cdots, N_{T}}\left|X_{\tau_{j}}\right|$. For the simplicity of notation, we denote $j_{n}=\left[n /\left(k_{n}-1\right)\right]$ in the following. For small $\Delta_{n}$, we have that a.s. in any time interval $\left(t_{i}, t_{i+k_{n}-1}\right]$, at most only one jump can occur. Moreover, we can obtain that the pre-averaging observation $\bar{Z}_{0 i}^{n}$ of continuous diffusion process without jump satisfies $\sup \bar{Z}_{0 i}^{n}=O\left(\Delta_{n}^{1 / 4} \log 1 / \Delta_{n}\right)$, while the pre-averaging observation of jump process $\bar{J}_{i}^{n}$ is greater than $\bar{X}$ multiplying some constant, which is not negligible. So we propose the following estimator for jump size $X_{\tau^{(i)}}$,

$$
\hat{X}_{\tau^{(i)}}=\bar{Z}_{i}^{n} I_{\left\{\left(\bar{Z}_{i}^{n}\right)^{2}>r\left(\Delta_{n}\right)\right\}} .
$$

Yu et al. [19] demonstrated the theoretical properties of estimator (4). The results shows that for each $i, \hat{X}_{\tau^{(i)}}$ estimates the product of some constant $\bar{g}$ and the size of the first jump occurs within $\left(t_{i}, t_{i+k_{n}-1}\right], \bar{g} X_{\tau^{(i)}} I_{\left\{i_{i}^{i+k_{n}-1} N \geq 1\right\}}$, where 
$\bar{g}=\int_{0}^{1} g(s) \mathrm{d} s$.

\subsection{Intraday Jump Tail Risk Measurement}

In this subsection, we present how to model the intraday jump tail and then to measure the jump tail risk, i.e. VaR (Value-at-Risk) and ES (Expected Shortfall) based on extreme value theory (EVT). Extreme value theory provides simple parametric models to capture the extreme tails of distribution and to forecast risk. There are mainly two methods of applying EVT: the first is known as the Block Maxima (Minima) (BMM) method based on the generalized extreme value distribution (GEV), while the second is known as the peaks-over-threshold (POT) approach based on the generalized Pareto distribution (GPD). Since the POT method uses GPD to fit the exceedances over a given threshold and hence it doesn't require a large data set as BMM, it is considered more efficient in modelling limited data (McNeil, Frey and Embrecht [21]). Thus, in the following, we use the POT method to model the tail distribution of the identified intraday jump series.

Suppose that the jump series $\left\{X_{i}\right\}$ are identically distributed random variables with unknown underlying distribution function $F(x)=P\left(X_{i} \leq x\right)$. The excess distribution $F_{u}$ over a threshold $u$ is given by

$$
F_{u}(y)=P(X-u \leq y \mid X>u)=\frac{F(y+u)-F(u)}{1-F(u)}=\frac{F(x)-F(u)}{1-F(u)}
$$

for $0<y<x_{F}-u$, where $x_{F} \leq \infty$ is the right endpoint of $F$, and $y=x-u$.

In EVT framework, there is a key result that for a large class of underlying distributions $F$ (containing all the common continuous distributions in statistics, such as normal, lognormal, t, gamma, exponential, beta, etc.), as the threshold $u$ progressively increases, the excess distribution $F_{u}$ converges to a generalized Pareto distribution. In the sense of this result, the GPD is the natural model for the excess distribution above sufficiently high thresholds. That is the excess distribution function $F_{u}$ can be approximated by GPD for a certain $u$ :

$$
F_{u}(y) \approx G_{\xi, \sigma}(y),
$$

where $G_{\xi, \sigma}$ is the generalized Pareto distribution (GPD), which is given by

$$
G_{\xi, \sigma}(y)=\left\{\begin{array}{lll}
\left(1+\frac{\xi}{\sigma} y\right)^{-1 / \xi} & \text { if } & \xi \neq 0 \\
1-e^{-y / \sigma} & \text { if } & \xi \neq 0
\end{array}\right.
$$

for $y \in\left[0,\left(x_{F}-u\right)\right]$ if $\xi \geq 0$, and $y \in\left[0,-\frac{\sigma}{\xi}\right]$ if $\xi<0$. Here $\xi$ is the shape parameter and $\sigma$ is the scale parameter for GPD.

Hence, for $x \geq u$, replacing the $F_{u}$ by GPD,

$$
\begin{aligned}
\bar{F}(x) & =P(X>u) P(X>x \mid X>u) \\
& =\bar{F}(u) P(X-u>x-u \mid X>u) \\
& =\bar{F}(u) \bar{F}_{u}(x-u)=\bar{F}(u)\left(1+\xi \frac{x-u}{\sigma}\right)^{-1 / \xi} .
\end{aligned}
$$


This gives a formula for tail probabilities. The inverse of (8) gives the high quantile of the distribution or VaR. Thus, for $\alpha \geq F(u)$ (i.e. tail probability is $1-\alpha), \mathrm{VaR}$ is given by

$$
\operatorname{VaR}_{\alpha}=q_{\alpha}(F)=u+\frac{\sigma}{\xi}\left(\left(\frac{1-\alpha}{\bar{F}(u)}\right)^{-\xi}-1\right) .
$$

For $\xi<1$, the $\mathrm{ES}$ is given by

$$
E S_{\alpha}=\frac{1}{1-\alpha} \int_{\alpha}^{1} q_{x}(F) \mathrm{d} x=\frac{V a R_{\alpha}}{1-\xi}+\frac{\sigma-\xi u}{1-\xi} .
$$

Equations (9) and (10) give the theoretical formulae to calculate the jump tail risk measure. In the following, we show that how to estimate the VaR and ES with the identified jump series.

For the identified jump series $\left\{\hat{X}_{i}\right\}$, if there are total $n$ observations and $N_{u}$ of observations above $u$, we get an empirical estimator $N_{u} / n$ of $\bar{F}(u)$. Putting the maximum likelihood estimates of the parameters of the GPD together, we arrive an estimator for tail distribution $F(x)$,

$$
\hat{F}(x)=1-\frac{N_{u}}{n}\left(1+\frac{\hat{\xi}}{\hat{\sigma}}(x-u)\right)^{-1 / \hat{\xi}} .
$$

Also, we get the estimator of $\operatorname{VaR}$

$$
\widehat{V a R}_{\alpha}=u+\frac{\hat{\sigma}}{\hat{\xi}}\left(\left(\frac{n}{N_{u}}(1-\alpha)\right)^{-\hat{\xi}}-1\right),
$$

and the estimator of $E S$

$$
\widehat{E S}_{\alpha}=\frac{\widehat{V a R}_{\alpha}}{1-\hat{\xi}}+\frac{\hat{\sigma}-\hat{\xi} u}{1-\hat{\xi}} .
$$

The estimation procedure presented above depends heavily on the important parameter $u$. In this paper, we will use the mean excess plot to choose a reasonable threshold. The idea behind this method is demonstrated as follows. Given a high threshold $u_{0}$, suppose that the excess $X-u_{0}$ follows a GPD with parameter $\xi$ and $\sigma$. Then the mean excess over the threshold $u_{0}$ is

$$
E\left(X-u_{0} \mid X>u_{0}\right)=\frac{\sigma}{1-\xi} .
$$

For any $u>u_{0}$, define the mean excess function $e(u)$ as

$$
e(u)=E(X-u \mid X>u)=\frac{\sigma+\xi\left(u-u_{0}\right)}{1-\xi} .
$$

Thus, for a fixed $\xi$, the mean excess function is a linear function of $u$ for $u>u_{0}$. This result leads to simple graphical method to infer the appropriate threshold value $u_{0}$ for the GPD. Define the empirical mean excess function as

$$
\hat{e}(u)=\frac{1}{N_{u}} \sum_{i=1}^{N_{u}}\left(x_{i}-u\right) \text {. }
$$

The scatter plot of $\hat{e}(u)$ against $u$ is called the mean excess plot, which 
should be linear in $u$ for $u>u_{0}$. Hence, we can choose a reasonable threshold according to the mean excess plot.

\section{Empirical Example}

In this section, we implement our procedure of measuring the intraday jump tail risk with actual high frequency data. We collect the transaction data for Microsoft Corporation (MSFT) shares carried out on NASDAQ from Jan 3, 2011 to Jul 29, 2011 from Wharton Research Data Services (WRDS). We use every ten seconds data to identify and estimate the intraday jumps in one minute return by implementing pre-averaging step with $k_{n}=7$ observations. Over this seven months time period, there were total 336,960 ten-seconds observations corresponding to daily 6.5 trading hours in valid 144 trading days excluding weekends and holidays. The return is calculated by $r_{t_{i}}=\left(\log P_{t_{i}}-\log P_{t_{i-1}}\right) \times 100$, where $P_{t_{i}}$ denotes the transaction price at $t_{i}$.

Firstly, we use the pre-averaging threshold method to estimate the intraday jump. Let $g(x)=x \wedge(1-x)$, which is used in Jacod et al. [17]. In addition, choose the threshold function following the studies in Christensen et al. [22]. In order to study the intraday dynamic pattern of jumps, we summarize their frequencies at one-minute frequency of all trading days. Figure 1 presents the frequency distribution of the identified intraday jumps occurred in 6.5 trading hours. It's obvious that the intraday jumps for MSFT from Jan 3, 2011 to Jul 29, 2011 take on "L"-type dynamics. It says that most jumps occurred around the market opening time. For example, there are over 40 trading days with jumps observed at 9:31 (i.e. one minute after the market opening). However, there are less than 10 trading days with jumps observed at half an hour after opening time. This "L"-type intraday pattern may be driven by the accumulations of news arrivals overnight.

Figure 2 presents the Q-Q plot of the estimated intraday jumps. The result shows that the intraday jump has fatter tails than normal distribution. This further demonstrates the reasonability of using the EVT to model the jump tails.

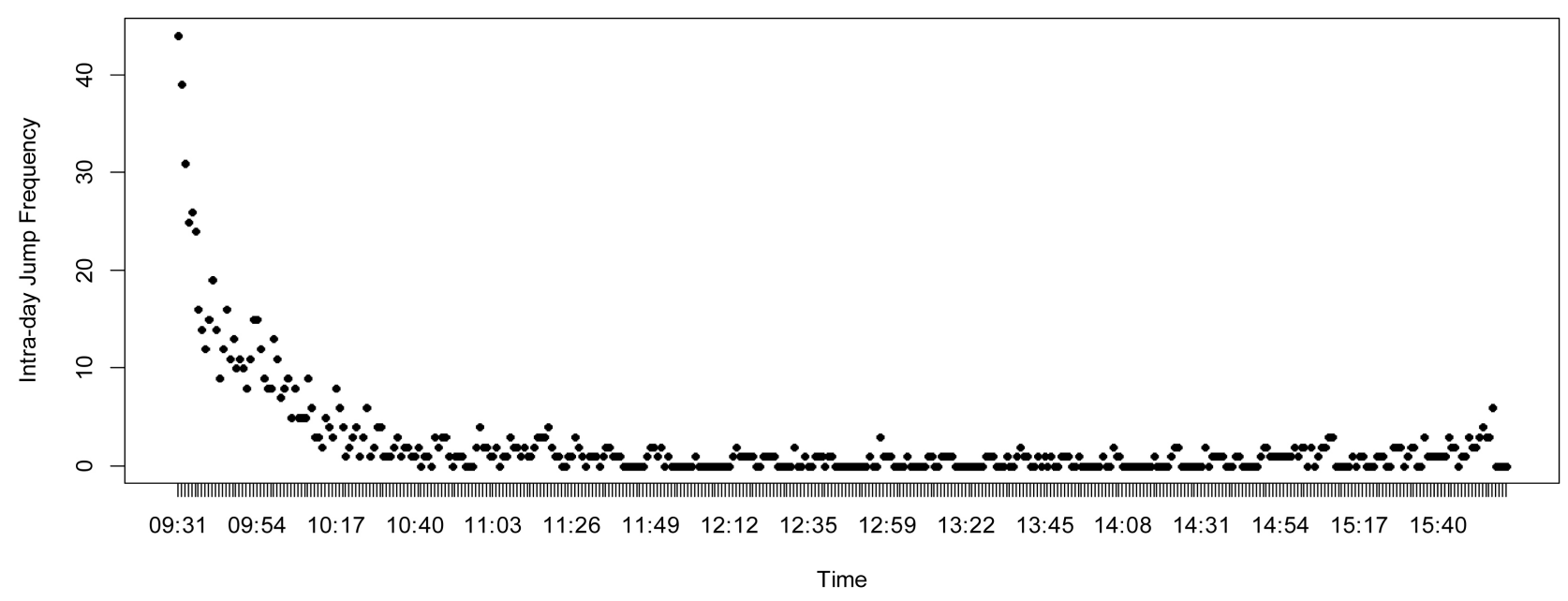

Figure 1. Frequency distribution of intraday jump. 
Next, we use the POT method and generalized Pareto distribution (GPD) to fit the negative and positive jump tail respectively. The threshold $u$ is chosen by the mean excess function. Figure 3 and Figure 4 present the mean excess plot for negative jump tail and positive jump tail respectively. Observing the plots, we choose $u=0.20$ for negative jump, and $u=0.25$ for positive jump.

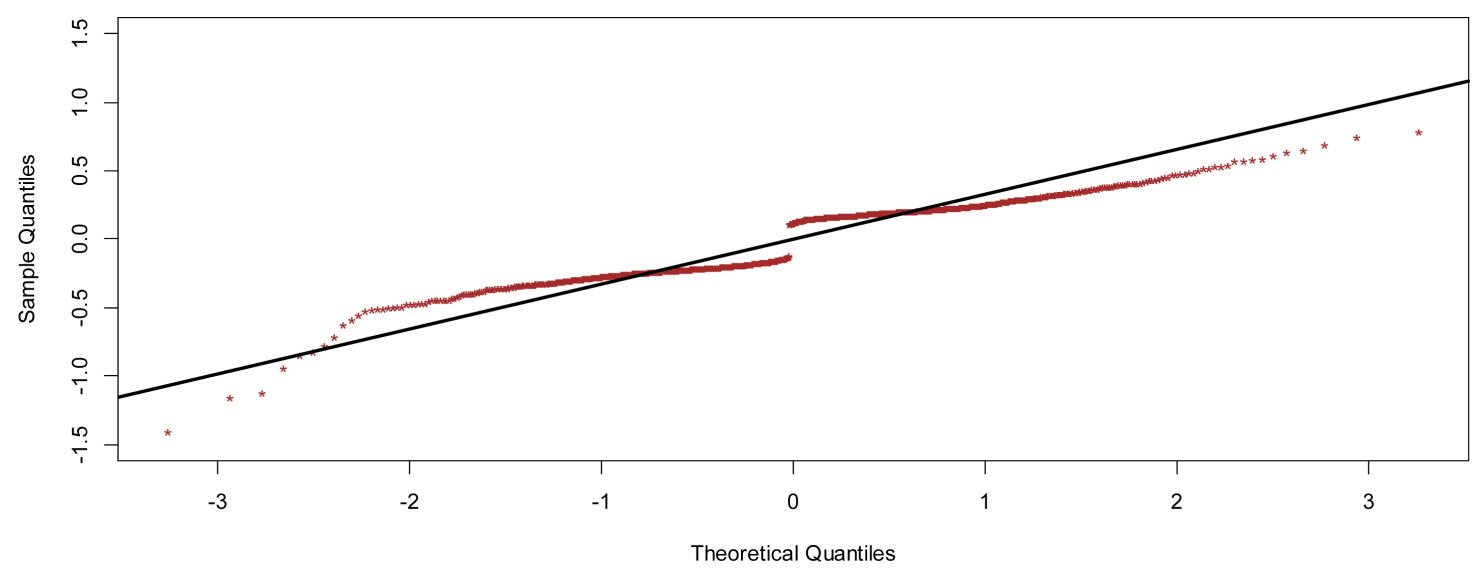

Figure 2. QQ plot of intraday jump.

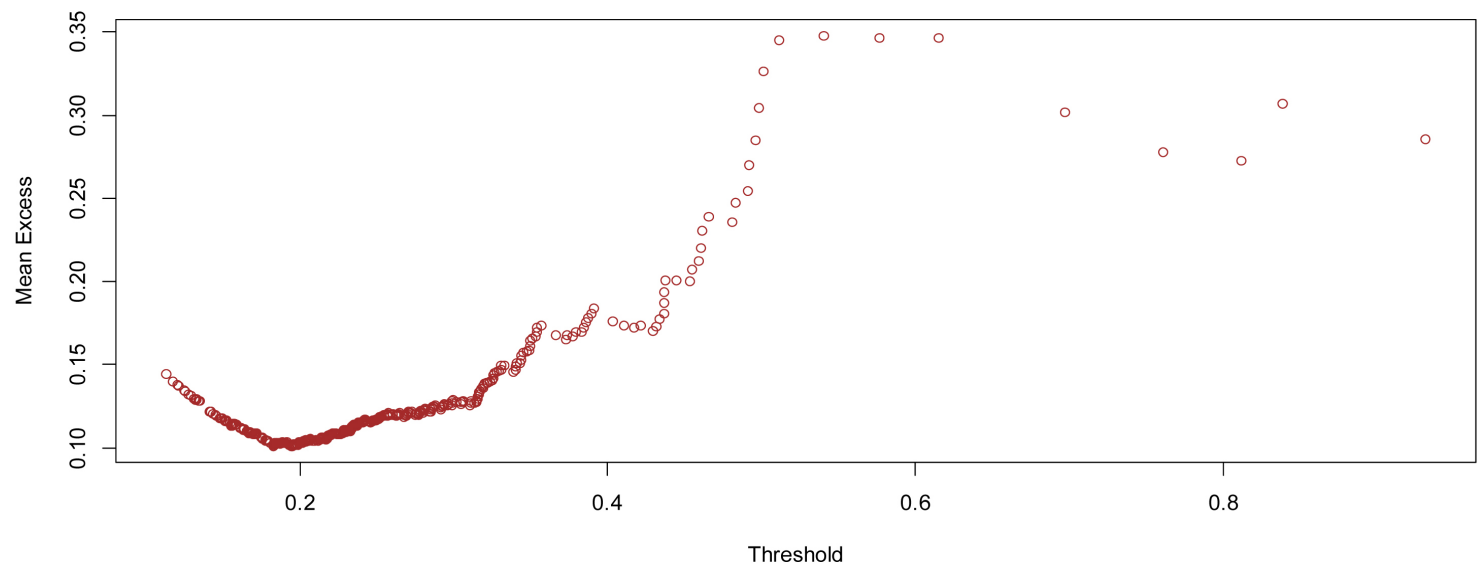

Figure 3. Mean excess function for negative jump tail.

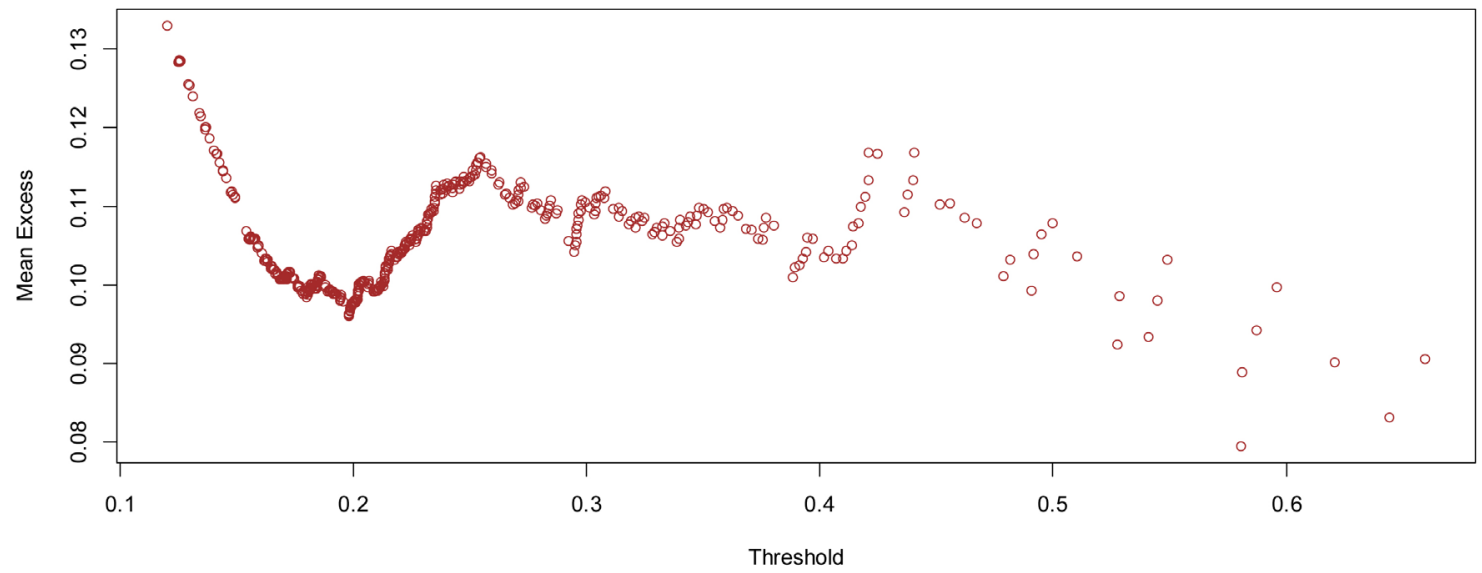

Figure 4. Mean excess function for positive jump tail. 
Based on the chosen threshold $u$, Table 1 presents the estimation results of intraday jump and jump tail. Firstly, we can see that there are 452 positive jumps and 437 negative jumps happened among the total one-minute return observations and the corresponding percentage is $0.81 \%$ and $0.78 \%$ respectively. The number of exceedances over threshold is 293 and 286 for positive and negative jump respectively. It seems that the number of jumps occurred or the intensity of jumps is symmetric for positive and negative jumps. Secondly, by comparing the results of jump tail distribution, we find that the shape parameter $\xi$ for positive jump is -0.0803 and is not significant at the given $10 \%, 5 \%, 1 \%$ levels, which means that positive jump tail may follow exponential distribution. However, the shape parameter $\xi$ for negative jump is 0.2176 and is significant at $1 \%$ level, which means that negative jump tail follows GPD with heavy tail. These results show that the positive and negative jump tail is asymmetric. In particular, the negative tail is heavier than the positive tail, which shows that there are more negative extreme events happened than positive events over the periods from Jan. 3, 2011 to Jul. 29, 2011 for MSFT.

We then calculate the $\mathrm{VaR}$ and ES for negative and positive jumps based on the above estimation results of jump tail distribution. The results of VaR and ES are presented in Table 2. We find that as the significance level (i.e. tail probability) decreases, the results of $\mathrm{VaR}$ and ES for negative jump becomes larger than positive jump as expected, which further demonstrates the asymmetry of negative and positive jump tails. Meanwhile, the values in parenthesis in Table 2 are the $p$ values in testing the validity of $V a R s$ and $E S$ s by Kupiec test. Values smaller than a given significance level indicate that the risk measures are invalid. From the results, we can see that the risk measure are valid except the case of $10 \%$ significance level for positive jump, which further in turn shows the success of our measuring method for jump tail risk.

\section{Conclusion}

Jump component in asset price process is a very important source of financial

Table 1. Estimation results of intraday jump and jump tail.

\begin{tabular}{ccc}
\hline & Negative jump & Positive jump \\
\hline Counts & 437 & 452 \\
Percentage & $0.78 \%$ & $0.81 \%$ \\
Threshold $u$ & 0.20 & 0.25 \\
Counts of exceedances & 286 & 293 \\
Percentage of exceedances & $65.45 \%$ & $64.82 \%$ \\
$\xi$ (shape parameter) & $0.2176^{* * *}$ & -0.0803 \\
& $(0.0669)$ & $(0.0796)$ \\
$\sigma$ (scale parameter) & $0.0809^{* * *}$ & $0.1225^{* * *}$ \\
& $(0.0071)$ & $(0.0138)$ \\
\hline
\end{tabular}

Note: Values in parenthesis are the standard errors of the estimates, ${ }^{*}{ }^{* *},{ }^{* *}$ mean that the results are significant at $10 \%, 5 \%, 1 \%$ level respectively. 
Table 2. Results of VaR and ES for intraday jump.

\begin{tabular}{ccccc}
\hline \multirow{2}{*}{ Sig. level } & \multicolumn{2}{c}{ Negative tail } & \multicolumn{2}{c}{ Positive tail } \\
\cline { 2 - 5 } & VaR & ES & VaR & ES \\
\hline $5.00 \%$ & 0.5417 & 0.5787 & 0.5762 & 0.6095 \\
& $(0.9654)$ & $(0.9177)$ & $(0.9955)$ & $(0.7538)$ \\
\multirow{2}{*}{$1.00 \%$} & 0.8408 & 0.8521 & 0.7216 & 0.7296 \\
& $(0.9795)$ & $(0.9795)$ & $(0.7815)$ & $(0.7815)$ \\
$0.50 \%$ & 1.0057 & 1.0124 & 0.7787 & 0.7829 \\
& $(0.9854)$ & $(0.9854)$ & $(0.6139)$ & $(0.6139)$ \\
$0.10 \%$ & 1.4994 & 1.5014 & 0.8995 & 0.9004 \\
& $(0.3405)$ & $(0.3405)$ & $(0.2977)$ & $(0.2977)$ \\
$0.01 \%$ & 2.5864 & 2.5867 & $1.0473^{*}$ & $1.0474^{*}$ \\
& $(0.1108)$ & $(0.1108)$ & $(0.0962)$ & $(0.0962)$ \\
\hline
\end{tabular}

Note: Values in parenthesis are the $p$ values in testing the validity of VaRs and ESs, ${ }^{*}{ }^{* *},{ }^{* *}$ mean that the risk measures are invalid at $10 \%, 5 \%, 1 \%$ level respectively.

extreme risk. With the availability of high frequency data, it has aroused wide attention of researchers in last two decades. However, with the frequency of data increases, the identification of jump and its relevant studies will run into the bias problem caused by market microstructure noise. In this paper, we propose a simple nonparametric method to identify the intraday jump and measure the intraday jump tail risk with noisy high frequency data. We use a two-step procedure to measure the jump tail risk. In first step, we use a pre-averaging approach to diminish the effects of noises, and then propose the pre-averaging threshold estimator of intraday jump. In second step, we fit the tail distribution of the identified jump series with POT method and GPD, and then to calculate the risk measure (VaR and ES) of jump tail. Finally, we show the power of our procedure by a real data study. The results show that our proposed procedure of measuring the jump tail risk is valid and is easy to implement. Moreover, the nonparametric identification of intraday jump can also be used to analyze the dynamics of intraday jump, which is useful to study the microstructure of the market. Further studies on risk management, such as analyzing the impactors of jump tail risk, dynamic jump tail risk forecasting are the future research directions.

\section{Acknowledgements}

This research was supported in part by the NSFC (71601048), and the Fundamental Research Funds for the Central Universities in UIBE (13QD09).

\section{References}

[1] Mandelbrot, E. (1963) The Variation of Certain Speculative Prices. Journal of Business, 35, 394-419. https://doi.org/10.1086/294632

[2] Fama, E. (1965) The Behavior of Stock Market Prices. Journal of Business, 38, 34-105. https://doi.org/10.1086/294743 
[3] Barndorff-Nielsen, O.E. and Shephard, N. (2004) Power and Bipower Variation with Stochastic Volatility and Jumps. Journal of Financial Econometrics, 2, 1-37. https://doi.org/10.1093/jjfinec/nbh001

[4] Barndorff-Nielsen, O.E. and Shephard, N. (2006) Econometrics of Testing for Jumps in Financial Economics using Bipower Variation. Journal of Financial Econometrics, 4, 1-30. https://doi.org/10.1093/jjfinec/nbi022

[5] Huang, X. and Tauchen, G. (2005) The Relative Contribution of Jumps to Total Price Variance. Journal of Financial Econometrics, 3, 456-499. https://doi.org/10.1093/jifinec/nbi025

[6] Aït-Sahalia, Y. and Jacod, J. (2009) Testing for Jumps in a Discretely Observed Process. Annals of Statistics, 37, 184-222. https://doi.org/10.1214/07-AOS568

[7] Lee, S.S. and Hannig, J. (2010) Detecting Jumps from Lévy Jump Diffusion Processes. Journal of Financial Economics, 96, 271-290. https://doi.org/10.1016/j.jfineco.2009.12.009

[8] Lee, S.S. and Mykland, P.A. (2012) Jumps in Equilibrium Prices and Market Microstructure Noise. Journal of Econometrics, 168, 396-406.

https://doi.org/10.1016/j.jeconom.2012.03.001

[9] Bollerslev, T. and Todorov, V. (2011) Tails, Fears and Risk Premia. Journal of Finance, 66, 2165-2211. https://doi.org/10.1111/j.1540-6261.2011.01695.x

[10] Bollerslev, T. and Todorov, V. (2011) Estimation of Jump Tails. Econometrica, 79, 1727-1783. https://doi.org/10.3982/ECTA9240

[11] Bollerslev, T., Todorov, V. and Li, S.Z. (2013) Jump Tails, Extreme Dependencies, and the Distribution of Stock Returns. Journal of Econometrics, 172, 307-324.

https://doi.org/10.1016/j.jeconom.2012.08.014

[12] O’Hara, M. (1995) Market Microstructure Theory. Blackwell, Cambridge.

[13] Hasbrouck, J. (2004) Lecture Notes for Ph.D. Seminar in Empirical Market Microstructure. New York University, New York.

[14] Zhang, L., Mykland, P.A. and Aït-Sahalia, Y. (2005) A Tale of Two Time Scales: Determining Integrated Volatility with Noisy High-Frequency Data. Journal of the American Statistical Association, 100, 1394-1411. https://doi.org/10.1198/016214505000000169

[15] Zhang, L. (2006) Efficient Estimation of Stochastic Volatility Using Noisy Observations: A Multi-Scale Approach. Bernoulli, 12, 1019-1043.

https://doi.org/10.3150/bj/1165269149

[16] Podolskij, M. and Vetter, M. (2009) Estimation of Volatility Functionals in the Simultaneous Presence of Microstructure Noise and Jumps. Bernoulli, 15, 634-658. https://doi.org/10.3150/08-BEJ167

[17] Jacod, J., Li, Y.Y. and Mykland, P.A. (2009) Microstructure Noise in the Continuous Case: The Pre-Averaging Approach. Stochastic Processes and Their Applications, 119, 2249-2276. https://doi.org/10.1016/j.spa.2008.11.004

[18] Barndorff-Nielsen, O.E., Hansen, P.R., Lunde, A. and Shephard, N. (2008) Designing Realised Kernels to Measure Ex-post Variation of Equity Prices in the Presence of Noise. Econometrica, 76, 1481-1536. https://doi.org/10.3982/ECTA6495

[19] Yu, C., Zhao X.J. and Zhang, B. (2015) Nonparametric Estimation of Jump Characteristics under Market Microstructure Noise. Communications in Statistics-Simulation and Computation, 1-13.

[20] Jing, B.Y., Liu, Z. and Kong, X.B. (2014) On the Estimation of Integrated Volatility with Jumps and Microstructure Noise. Journal of Business and Economic Statistics, 32, 457-467. https://doi.org/10.1080/07350015.2014.906350 
[21] McNeil, A.J., Frey, R. and Embrechts, P. (2005) Quantitative Risk Management: Concepts, Techniques and Tools. Princeton University Press, Princeton.

[22] Christensen, K, Oomen, R. and Podolskij, M. (2014) Fact or Friction: Jumps at Ultra-High Frequency. Journal of Financial Economics, 114, 576-599.

https://doi.org/10.1016/j.jfineco.2014.07.007

Submit or recommend next manuscript to SCIRP and we will provide best service for you:

Accepting pre-submission inquiries through Email, Facebook, LinkedIn, Twitter, etc. A wide selection of journals (inclusive of 9 subjects, more than 200 journals)

Providing 24-hour high-quality service

User-friendly online submission system

Fair and swift peer-review system

Efficient typesetting and proofreading procedure

Display of the result of downloads and visits, as well as the number of cited articles Maximum dissemination of your research work

Submit your manuscript at: http://papersubmission.scirp.org/

Or contact ojs@scirp.org 